\title{
Agôn
}

Revue des arts de la scène

HS 1 | 2011

Mettre en scène l'événement

\section{«Vérité » ou « mensonge »? Le Théâtre politique post-11 septembre en Grande-Bretagne}

\section{Clare Finburgh}

\section{(2) OpenEdition \\ Journals}

Édition électronique

URL : http://journals.openedition.org/agon/1808

DOI : 10.4000/agon. 1808

ISSN : 1961-8581

Éditeur

Association Agôn

Référence électronique

Clare Finburgh, « « Vérité » ou « mensonge » ? Le Théâtre politique post-11 septembre en GrandeBretagne », Agôn [En ligne], HS 1 | 2011, mis en ligne le 05 septembre 2011, consulté le 17 avril 2020. URL : http://journals.openedition.org/agon/1808; DOI : https://doi.org/10.4000/agon.1808 


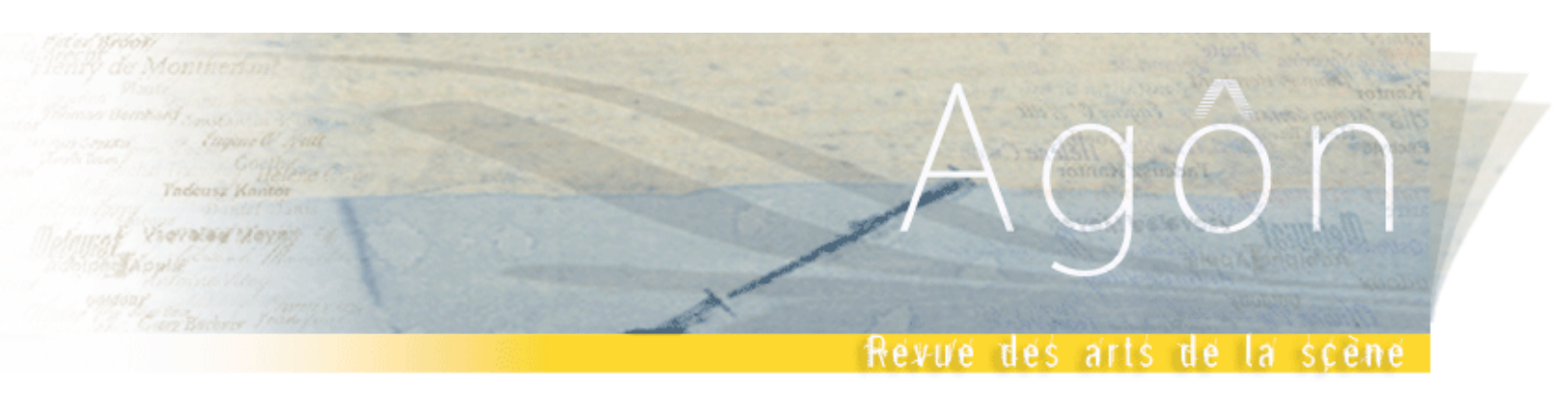

\title{
"Vérité » ou «mensonge »? Le Théâtre politique post-11 septembre en Grande-Bretagne ${ }^{1}$
}

\author{
Clare FinBURGH
}

Les conséquences des attentats terroristes du 11 septembre 2001 se sont ressenties jusque dans le théâtre britannique. Depuis une dizaine d'années, en Grande-Bretagne, le théâtre politique se développe considérablement. Michael Billington, correspondant théâtral du Guardian, quotidien britannique de gauche, explique :

Deux facteurs ont conduit à la réinvention du théâtre politique : le public en a eu marre de voir le New Labour accorder plus d'importance à son image qu'aux vrais problèmes, et il a été consterné par la politique étrangère du couple Bush-Blair. Quel que soit le verdict que l'Histoire portera sur les années Blair, ce qui est sûr c'est que celles-ci ont réactivé le théâtre politique. $^{2}$

Les attentats du 11 septembre 2001, fomentés, semble-t-il, par Ousama ben Laden et par les Talibans afghans, ont déclenché dans l'administration du Président George W. Bush la Guerre contre le terrorisme, politique agressive contre tout ennemi potentiel des États-Unis. Un an après les attentats, Bush cible l'Irak de Saddam Hussein, qu'il accuse de soutenir des groupes terroristes, et qu'il perçoit comme une menace immédiate. En avril 2002 à Crawford (Texas), dans le ranch de Bush, Tony Blair, Premier ministre britannique, promet au Président de le soutenir en cas d'une invasion de l'Irak. Blair rentre en Grande-Bretagne, où il assure à son gouvernement et ses électeurs de n'avoir signé aucun pacte avec les ÉtatsUnis. Dans ce climat de tromperie causé par la Guerre contre le terrorisme, "le théâtre politique, selon Billington, devient une nécessité vitale plus qu'un luxe » (Billington, p. 384).

Que signifie le terme «théâtre politique »? À certains égards, tout théâtre, comme tout art, est politique : tout théâtre peut s'écrire et se lire d'un point de vue idéologique. Depuis une décennie, pourtant, la politique occupe une place centrale, frontale et explicite dans le théâtre britannique. Ceci dit, le théâtre politique britannique n'est en rien un genre uniforme et monolithique. Ce théâtre comprend une variété considérable de styles et d'approches. Drunk Enough to Say I Love You de Caryl Churchill (Assez saoul pour te dire que je t'aime, Royal Court Theatre, 2006) est une allégorie onirique ou cauchemardesque de la special relationship (relation privilégiée) entre les États-Unis et la Grande-Bretagne ; Product de Mark Ravenhill (Produit, Traverse Theatre, 2005) est une satire de l'exploitation faite par Hollywood de l'horreur des attentats ; Days of Significance de Roy Williams (Jours

\footnotetext{
1 Je tiens à remercier Christophe Brault pour son écoute inappréciable et sa relecture attentive.

${ }^{2}$ New Labour est le nom attribué au parti travailliste du Premier ministre Tony Blair (1996-2007). L'auteure est la responsable des traductions qui suivent.
} 
d'importance, Royal Shakespeare Company, Swan Theatre, 2007) est un récit factuel sur le racisme des troupes britanniques en Irak; Iphigenia at Aulis d'Euripide mise en scène par Katie Mitchell (National Theatre, 2004) devient un débat sur les sacrifices que ferait un chef d'État pour gagner une guerre ; Extreme Rambling : Walking the Wall (Tricycle Theatre, 2011) est un one-man show du comique Mark Thomas, sur le mur érigé par Israël entre ses territoires et ceux des Palestiniens ${ }^{3}$. Le théâtre politique britannique est donc éminemment varié. Je me concentrerai ici sur un genre qui, depuis dix ans, occupe le devant de la scène, pour ainsi dire : le théâtre verbatim.

Qu'est-ce que le théâtre verbatim ? Dans cet article j'élaborerai une définition de ce genre, tout en évaluant ses mérites et ses limites.

\section{Le devoir d'enquête. Un théâtre au plus près du réel}

Une pièce verbatim se compose - de manière générale - de paroles prononcées par des personnes réelles et citées mot à mot. Ces individus sont interviewés, et leur discours est enregistré et transcrit. L'auteur arrange, agence, aménage leurs paroles, afin de fabriquer une forme dramatique qui permettra à des acteurs de les prononcer. Toutefois, les pièces verbatim peuvent varier énormément (Hammond et Steward, p. 9). La pièce qui peut être considérée comme la genèse du théâtre verbatim, L'Instruction de Peter Weiss (1965), est une élégie poétique : issues des procès de Francfort (1963-65), les attestations des anciens gardes du camp nazi d'Auschwitz, et des survivants, sont composées sous forme de « Cantos »-scènes versifiées inspirés de l'Inferno de Dante. Inversement, les tribunal plays du Tricycle Theatre, auxquelles je consacrerai cet article, tâchent d'omettre toute intervention artistique de la part de l'auteur et du metteur en scène.

Depuis 1984, Nicolas Kent dirige le Tricycle, petit centre culturel de Londres qui est considéré comme le premier théâtre politique de Grande-Bretagne. L'intérêt de Kent pour l'actualité et pour la justice sociale influence nettement sa programmation. Half the Picture (La Moitié du tableau, 1993), écrite par l'auteur écossais le plus puissant du théâtre politique des années soixante-dix et quatre-vingt, John McGrath, et le rédacteur spécialiste des problèmes de sécurité du Guardian, Richard Norton-Taylor, a été la première pièce verbatim créée au Tricycle. Elle présente la Scott Inquiry, enquête faite sur la vente d'armes à l'Irak par des compagnies britanniques pendant l'embargo. Elle est la première d'une longue série de tribunal plays - pièces dont la matière brute provient d'enquêtes judiciaires ${ }^{4}$. Chacune des tribunal plays est suivie d'un débat, souvent animé par une figure politique ${ }^{5}$. En outre, le public de ces pièces se compose souvent de ministres, de diplomates étrangers et d'autres figures politiques, qui viennent s'informer sur un sujet d'actualité. Depuis les attentats de 2001, Norton-Taylor et Kent créent plusieurs pièces verbatim sur le terrorisme et sur les guerres qui s'ensuivent. Mon analyse se resserrera autour de deux de ces pièces : Justifying War: Scenes from the Hutton Inquiry (Justifier la guerre : scènes de l'enquête Hutton, 2004) et Called to Account: The Indictment of Anthony Charles Lynton Blair for the Crime of

\footnotetext{
${ }^{3}$ Pour plus de détails sur la représentation du terrorisme et de la guerre dans le théâtre britannique, voir les deux articles écrits par Finburgh.

4 Nuremberg (1996) est basée sur les témoignages du Procès de Nuremberg intenté contre les chefs de l'Allemagne nazie ; Srebrenica (1998) est fait des transcriptions du procès international sur la guerre en Bosnie ; The Colour of Justice (La Couleur de la justice, 1999) inclut des témoignages de l'enquête sur le traitement par la police du meurtre d'un jeune noir ; Bloody Sunday: Scenes from the Saville Inquiry (Dimanche sanglant, 2005) est tirée de l'enquête sur les manifestants irlandais tués par les militaires britanniques en 1972.

${ }^{5}$ Par exemple Menzies Campbell, député Liberal Democrat, ou Tony Benn, ancien ministre Labour.
} 
Aggression Against Iraq (Appelé à rendre des comptes : la mise en examen d'Anthony Charles Lynton Blair pour le crime d'aggression de l'Irak, 2007).

Comment expliquer ce besoin, aujourd'hui, d'un théâtre factuel ? Tout d'abord, la méfiance vis-à-vis du journalisme est de plus en plus répandue. Les médias - télévision, radio, journaux, internet - ne représentent plus une source fiable d'informations. NortonTaylor, lui-même non seulement auteur de théâtre verbatim, mais aussi journaliste, se plaint : « les coupes forcément brutales et inégales de la part des rédacteurs de presse ; la lutte constante pour l'espace dans les journaux et pour le temps d'antenne, sont des problèmes du journalisme » (Norton-Taylor (c), p. 121-2). Selon nombre de journalistes, les médias n'accordent presque plus de temps ni de place au journalisme d'investigation. Les médias sont sur-saturés de services d'informations permanentes, 24 heures sur 24, mais elles souscommuniquent les vrais détails des faits. Par exemple, les enquêtes judiciaires sont très rarement diffusées à la radio et à la télévision. Lorsqu'elles paraissent dans la presse, seuls les détails les plus salaces et les scoops font la une (Norton-Taylor (c), p. 125, 129). Kent insiste donc sur la «nécessité » de faire au théâtre de la place pour les faits importants omis par les médias (Kent, p. 135-6).

D'une part, les médias simplifient et tronquent les comptes-rendus des événements. D'autre part, les enquêtes publiques, comme celles que présentent les tribunal plays, sont très complexes et durent souvent des semaines, voire des années. La majorité du public n'a ni le temps ni l'envie d'assister à une enquête judiciaire entière. La tâche du théâtre, selon Kent et Norton-Taylor, est de rendre l'investigation plus abordable. Ils passent en revue des milliers de pages de témoignages, afin de choisir les moments qui, pour eux, sont les plus importants. L'enquête dont provient Justifying War a duré 25 jours et a réuni 75 témoins. Kent et NortonTaylor ont condensé ceci en une pièce de deux heures ne comprenant plus que douze témoins. Le but des tribunal plays est donc d'apporter à un public non-expert les instants les plus marquants de l'enquête - les « highlights », pour citer Kent (Kent, p. 154).

Examinons deux tribunal plays créées dans le sillage des attentats du 11 septembre 2001. La Hutton Inquiry, dont Justifying War (2004) tire son matériau, a été menée par l'avocat Lord Hutton afin de déterminer les circonstances menant à la mort de Dr David Kelly, expert en armes. La liste des témoins comprend, entre autres, Andrew Gilligan (journaliste de la radio BBC), Dr Brian Jones (chef de la section scientifique des services de renseignements), Alastair Campbell (spécialiste en communication chargé de l'image du Conseil des ministres de Tony Blair, de 1997 à 2003), Geoff Hoon (ministre de la défense de 1999 à 2003), Patrick Lamb (membre du ministère des Affaires étrangères), Gavyn Davies (président de la BBC de 2001 à 2004), et Janice Kelly (l'épouse de David Kelly). L'enquête et la pièce - révèlent les événements suivants. En mai 2005, à une heure de grande écoute, Gilligan a présenté un reportage prétendant qu'un expert en armes des services de renseignements, qui souhaitait rester anonyme, a avancé que le gouvernement britannique a exagéré l'affirmation selon laquelle Saddam Hussein possédait des armes de destruction massive (ADM). Selon la source anonyme, la déclaration faite par le gouvernement en septembre 2002 (les ADM de Saddam seraient prêtes à être lancées en 45 minutes), était issue d'une thèse d'étudiant publiée sur Internet (source peu fiable)! Les services de renseignements ne voulaient pas que ce détail soit inclus dans la déclaration. Comme je l'ai déjà mentionné, depuis 2002 Blair s'était engagé dans la Guerre contre le terrorisme menée par Bush, dont la prochaine cible était l'Irak. Campbell, chargé de communication du gouvernement Blair, a donc insisté pour que la mention des 45 minutes soit incluse dans la déclaration, afin que les arguments en faveur de l'invasion de l'Irak paraissent, selon Gilligan, plus « sexed-up », ou vendeur. Le gouvernement était furieux que la BBC l'accuse d'exagération, et Campbell a demandé que soit révélé le nom de l'informateur. C'était le Dr David Kelly. Le gouvernement a riposté en disant qu'il ne s'agissait que d'un fonctionnaire 
subalterne dont l'opinion n'avait pas d'importance, et l'a interrogé de manière agressive. Quelques jours plus tard, on retrouva son cadavre.

La pièce Called to Account (2007) diffère de Justifying War en ce qu'elle n'est pas basée sur une enquête publique. Norton-Taylor a demandé à quatre avocats d'interroger une douzaine de témoins afin de déterminer si la Cour pénale internationale pouvait accuser Tony Blair du crime d' " agression » pour l'invasion de l'Irak (une véritable enquête a été ouverte en 2009, et est toujours en cours). L'enquête est donc fictionnelle, mais les témoins existent réellement : entre autres, Richard Perle (conseiller de Donald Rumsfeld, ancien ministre américain de la défense), Scott Ritter (expert en contrôle des armements pour les États-Unis et pour l'Organisation des Nations Unies), Clare Short (ministre britannique), et Michael Smith (journaliste). Les témoins répondent à quatre questions : l'objectif de Blair, en déclarant la guerre contre l'Irak, était-il l'élimination des ADM ou un changement de régime ? Blair s'est-il engagé à envahir l'Irak après la résolution de l'ONU de mars 2003, ou un an avant, au ranch de Bush ? A-t-il exagéré la preuve supposée que l'Irak possédait des ADM ? Savait-il qu'une telle agression contre l'Irak pouvait être illégale ? Les témoins révèlent successivement que Blair s'était engagé à la Guerre contre le terrorisme bien avant 2003, sans informer son gouvernement. Ritter et Perle confirment que l'administration Bush ne pensait pas que les inspecteurs de l'ONU allaient trouver des ADM en Irak (Norton-Taylor (b), p. 21, 78). Called to Account, comme Justifying War, montre donc que Blair a exagéré sur la question des ADM, afin de justifier une intervention à laquelle il avait déjà souscrit. Ces deux tribunal plays exposent les effets néfastes de la Guerre contre le terrorisme, déclarée après les événements du 11 septembre 2001, sur la politique britannique. D'ailleurs, elles prétendent fournir au public les éléments du débat qui seraient omis par les médias.

Selon Kent et Norton-Taylor, non seulement les médias ne fournissent que les détails les plus séduisants, mais, de plus, ils sont incapables d'objectivité. Dans Justifying War, Campbell assure ne pas avoir influencé le gros titre du journal l'Evening Standard : « 45 Minutes from Attack » (Norton-Taylor (b), p. 43), alors qu'on a appris par la suite qu'il l'avait effectivement fait (Norton-Taylor (c), p. 119-20). Les médias ont tendance à reproduire, sans la questionner, la fictionnalisation de la réalité produite par les gouvernements. David Hare, l'auteur dramatique britannique qui jouit du plus grand succès et qui a écrit de nombreuses pièces verbatim - notamment Stuff Happens (National Theatre, 2004) sur la Guerre contre le terrorisme - dit que le théâtre d'aujourd'hui remplit le vide laissé par un journalisme partial (Hare, p. 63). Et Billington raconte que «le public s'intéresse de plus en plus au théâtre, qui peut lui donner une information brute sur un événement » (Billington, p. 385). Les pièces verbatim sont censées raconter les événements avec plus de détail, plus d'exactitude, plus d' « objectivité », et moins de mensonges (Kent, p. 164).

Le style dans lequel les tribunal plays sont mises en scène par Kent colle avec son désir de représenter la "réalité absolue » de manière « objective », non-médiatisée. D’abord, le style du dialogue des tribunal plays est naturaliste. Les discours des témoins et des avocats sont transcrits mot pour mot. Par exemple, dans la première scène de Justifying War, Hutton dit :

Can I just ask you Mr Lamb on part 2 of the September dossier Dr Kelly would have commented on that, he did not actually write it, he commented on it, but he actually wrote it, did he, the first draft of the box on page 38 ? (Norton-Taylor (a), p. 11). ${ }^{6}$

\footnotetext{
${ }^{6}$ « Puis-je juste vous demander Mr Lamb la deuxième partie du dossier de septembre Dr Kelly l'aurait commentée, il ne l'a pas écrit, il l'a commenté, mais en effet il l'a écrit, n'est-ce pas, la première version page $38 ? »$
} 
Ici, la syntaxe est peu structurée, la grammaire peu orthodoxe. Et dans Called to Account, les fautes d'anglais faites par Dr Shirwan Al-Mufti, témoin irakien, ne sont pas corrigées par l'auteur. (Norton-Taylor (b), p. 12). Le paradoxe du langage dramatique est qu'il est écrit, travaillé et littéraire, alors que le comédien doit le faire passer pour la langue parlée, spontanée et quotidienne. Le théâtre, pour reprendre l'analyse de Pierre Larthomas, est « un compromis entre deux langages » (Larthomas, p. 25). Pourtant, dans le théâtre verbatim, le langage dramatique est tout sauf un faux langage parlé ; il est littéralement le langage parlé. D'ailleurs, dans les mises en scène des tribunal plays, cette langue verbatim est parlée par les acteurs de manière naturaliste, jusqu'aux hésitations et bégaiements. En outre, chaque acteur porte un micro discret, pour ne pas être obligé de projeter sa voix. En effet, toute mise en scène diffuse la théâtralité. Kent explique :

Dans une tribunal play, les effets dramatiques sont à éviter... Pour les acteurs, ce n'est pas comme une pièce comme les autres. Ils savent qu'ils prennent part à quelque chose qui est, dans une certaine mesure, $l^{\prime}$ « histoire ». Ils portent un tel sens de responsabilité par rapport à la vérité et par rapport au projet, que, dès que quelqu'un joue, tout le monde le sait - donc personne ne joue. C'est comme s'il y avait un code tacite: il ne faut pas rechercher l'effet. (Kent, p. 155-6).

Et Norton-Taylor décrit les acteurs comme des «messagers » qui doivent communiquer, de manière fidèle, la vérité d'un tel fait (Norton-Taylor (c), p. 128). L'hyper-naturalisme du jeu est complété par l'éclairage : le plateau, ainsi que la salle, sont inondés de lumière, afin de produire l'ambiance d'une salle d'audience. La mise en scène est donc sobre et simple, pour présenter de manière immédiate les paroles des témoins. Kent explique: «L'hypernaturalisme et l'ambiance discrète nous aident à nous rapprocher de la vérité » (Kent, 156).

Kent exige des acteurs de ne pas «jouer ». En même temps, ils sont censés imiter les personnages qu'ils représentent (je reviendrai plus tard sur cette contradiction potentielle). Kent encourage chaque acteur à faire des recherches sur son personnage, à lire sa déposition juridique, et même à le rencontrer, afin de connaître son accent, son ton, ses gestes (Kent, p. 158). En outre, il est évident que Kent distribue les rôles en tenant compte de l'aspect physique des acteurs. Par exemple, l'Irakien dans Called to Account, Mufti, a été joué par Raad Rawi, acteur d'origine arabe. Ensuite, les costumes et coiffures des acteurs copient méticuleusement ceux des personnages joués. Kent explique que, comme les tribunal plays sont présentées souvent en même temps que se déroulent les vrais événements, il est important que l'interprétation des personnages soit convaincante (Kent, p. 157).

Si Kent et Norton-Taylor veulent présenter les témoignages de la façon la plus sobre, non modifiée et « naturelle », pourquoi ne pas les publier dans une brochure ou en ligne ? Ils répondraient qu'il est essentiel que les tribunal plays soient jouées en public (Norton-Taylor (c), p. 125). Béatrice Picon-Vallin, théoricienne du théâtre, explique la valeur de la dimension collective et unificatrice du théâtre : "le metteur en scène est porteur d'une utopie de la société, puisqu'il rassemble autour de lui un groupe d'artistes qui doit, en présence d'un autre groupe qui est le public, devenir une communauté » (Picon-Vallin, p. 119). Les spectateurs écoutent, éprouvent, témoignent ensemble. Avec les tribunal plays, on accorde même un rôle au public. Justifying War commence lorsque l'Huissier demande: "Messieurs Dames, veuillez éteindre vos téléphones portables. » (Norton-Taylor (a), p. 9). Peu après, Hutton prie tout le monde de se dresser et de respecter deux minutes de silence pour Dr Kelly. Comme la majorité des Britanniques a été réellement émue par son suicide, les spectateurs ne jouent qu'à moitié cette commémoration. De façon similaire, Called to Account termine avec le discours de clôture de l'accusation et de la défense, comme si le public du théâtre était le jury. Tout cela produit plusieurs effets. Premièrement, les spectateurs partagent, en société, leur colère, leur joie, leur ennui. Ayant assisté à plusieurs de ces tribunal plays, je peux 
confirmer que des spectateurs sifflaient ou huaient pour déclarer leur dégoût, et applaudissaient pour communiquer leur approbation. Après tout, l'information dans ces pièces n'est pas fictionnelle; elle concerne de manière directe la vie publique des spectateurs. Deuxièmement, au lieu d'être lues sur une page, les paroles des témoins sont incarnées par des voix et des corps. Selon Alecky Blythe, autre auteur de théâtre verbatim, « entendre véritablement les paroles, de la façon dont elles ont été dites, brosse un tableau beaucoup plus vif que si on les lisait dans un journal»(Blythe, p. 101). Hare va plus loin dans sa revendication de la représentation « live » : « la presse est beaucoup plus manipulatrice que le théâtre. Je crois que la vérité de ce qui se passe est révélée lors du jeu théâtral, alors qu'elle n'est pas si clairement révélée lorsqu'elle est écrite sur la page. » (Hare, p. 71). Pour ces auteurs, le théâtre, plus que le journalisme, peut représenter l'esprit du témoin avec efficacité, vérité et honnêteté.

Il est clair que le Tricycle a pour but d'influencer le public de manière politique. L'intention des tribunal plays n'est pas de divertir le public; elle est de diffuser un message (Norton-Taylor (c), p. 128). Cet objectif ne serait-il pas vain, étant donné que $80 \%$ du public du Tricycle est déjà très informé des événements racontés dans les pièces ? Selon les statistiques de Kent, environ $15 \%$ des spectateurs arrivent indécis, et repartent persuadés. Il est encouragé par ce chiffre :

Ça vaut incontestablement la peine parce que $15 \%$ ça représente entre 30 et 40 personnes par soir dont nous avons changé l'opinion et qui ont appris quelque chose. Alors, c'est formidable - après tout, on peut assister à une réunion politique où il n'y a que cinq personnes (Kent, p. 146).

En outre, les créateurs des tribunal plays souhaitent avoir de l'influence au-delà du public du théâtre. L'une des tribunal plays, Nuremberg, a été la première pièce à être jouée au Parlement; Guantanamo a été présentée devant la Chambre des communes de Londres ainsi qu'au Congrès américain. Toutes les tribunal plays ont été diffusées ou bien à la télévision ou bien à la radio $\mathrm{BBC}$ et, selon le Tricycle, ont touché un public de plus de 30 millions de personnes à travers le monde. Les tribunal plays deviennent donc une arme dans la campagne contre l'injustice menée par le Tricycle.

Je viens de montrer à quel point les créateurs du théâtre verbatim, notamment ceux du Tricycle, veulent communiquer la " vérité » et la "réalité » des événements, avec, disent-ils, « objectivité », « immédiateté » et « fidélité ». Mais dans quelle mesure cette tâche est-elle possible et même souhaitable?

\section{De la dramaticité du théâtre verbatim}

Tout théâtre manipule, truque, dissimule la réalité qu'il représente; qu'il soit la représentation la plus expressionniste et métaphorique de la réalité (comme Rhinocéros de Ionesco et sa peinture du totalitarisme), ou la plus réaliste et directe (comme les tribunal plays du Tricycle). La mimèsis du jeu théâtral n'est pas la réalité d'un événement; l'acteur n'est pas la personne représentée. Qu'il s'agisse de mots, d'images ou de scènes, on ne peut parler du réel au théâtre qu'au prix de sa déréalisation. Que les créateurs du théâtre verbatim le veuillent ou non, leurs pièces sont des imitations, des représentations factices, des mises à distance, rejouées par ces mots, images et scènes.

La représentation met en ordre le chaos du monde. Même si les auteurs des tribunal plays tentent de suivre d'aussi près que possible les enquêtes qu'ils représentent, ils en manipulent nécessairement les données, afin d'établir une tension dramatique. Il est vrai qu'une enquête juridique en soi suit plus ou moins la dramaturgie classique : elle commence 
par une présentation des circonstances et des personnes concernées (l'exposition); il s'ensuit une série de témoignages représentant les différentes faces de l'argument (le conflit, ou le nœud), qui contient des révélations inattendues (la péripétie); et elle se termine par les discours de clôture (le dénouement) ${ }^{7}$. Dans la bonne tradition aristotélicienne, dont le théâtre classique s'inspire, les enquêtes ont un début, un milieu et une fin (Aristote, ch. 7). Une enquête judiciaire convient donc à un traitement dramatique. La grande différence entre une tragédie classique et une enquête est que, dans l'enquête, il n'y a pas de suspens : on sait d'avance qui est mort. Le spectateur britannique moyen savait que Kelly s'était suicidé ; et la pièce commence en expliquant que le but est d'enquêter sur sa mort. Norton-Taylor ne respecte pas tout à fait l'ordre des dépositions dans l'enquête, afin d'augmenter la tension dramatique dans Justifying War et d'y insérer du suspens. Dans le Hutton Inquiry, Janice Kelly avait déposé avant Dr Jones. Norton-Taylor, lui, change l'ordre des témoignages, pour que la pièce se termine avec les paroles de Mrs Kelly. Durant toute la pièce, les témoins rendent hommage à David Kelly et expliquent qu'il avait très mal pris le fait que le gouvernement l'ait traité de fonctionnaire « inférieur ». Dans la dernière scène, Mrs Kelly raconte :

Nous n'avons lu dans les journaux que quelques articles sur David. [...] il y avait plusieurs références à son statut modeste. Je ne sais pas si la réaction était plutôt la mienne ou la sienne mais il a été très sonné par ça. (Norton-Taylor (a), p. 88).

Un fil narratif se tisse le long de la pièce, menant à la dernière scène, où le public rencontre Mrs Kelly et ses révélations sur les derniers jours de son mari. Norton-Taylor rajoute donc du suspens, en aménageant l'ordre des scènes dans la pièce. Dans Called to Account, le récit va également crescendo. La pièce est divisée en deux actes. Norton-Taylor fait intervenir à la fin du premier acte le personnage le plus connu de la distribution, Clare Short, dont la démission du cabinet de Tony Blair avait été très discutée. Elle clôt sa scène en justifiant sa démission :

de la tromperie pure et simple [...] Des mensonges, des demi-vérités, des allusions, je crois vraiment que Tony, il ne se dit pas: « je vais dire un mensonge là », il est tellement - « je peux l'obtenir en faisant du charme, je peux juste raconter un peu ceci à Clare ou cela au cabinet et je peux me débrouiller », et il pense que c'est ça la politique. Il ne les perçoit pas comme des mensonges mais je suis désolée, ce sont des mensonges. (Norton-Taylor (b), p. 39).

Finir un acte sur une telle présentation d'un Premier ministre qui, à l'époque, était en exercice, est évidemment explosif.

Et comme dans un vrai drame théâtral, il est souvent possible de déceler dans les tribunal plays une signification «universelle» qui transcenderait la spécificité des faits historiques. Pour Hare, toute pièce verbatim doit fournir une métaphore qui apprend au public quelque chose au-delà des actualités ; la mort, l'amour, la souffrance, le sacrifice, le pouvoir, l'échec, etc. : «C'est une méprise totale du théâtre documentaire que de penser qu'il s'agisse tout simplement de présenter sur scène un ensemble de faits. » (Hare, p. 59). Norton-Taylor se fait l'écho de ce sentiment: "Les tribunal plays du Tricycle se proposent de fournir de l'information, d'expliquer, d'exposer, et de provoquer un débat. Mais elles abordent aussi des questions plus larges - l'honnêteté, la vérité et la responsabilité de ceux qui nous ont en leur pouvoir » (Norton-Taylor, p. 131). Les deux pièces abordées ici révèlent le fonctionnement $\mathrm{du}$ gouvernement britannique, des services de renseignements et de la $\mathrm{BBC}$, ainsi que leurs relations. Elles révèlent comment ces institutions sont capables de nier, de contredire,

\footnotetext{
${ }^{7}$ Pour les composantes du théâtre classique, voir Scherer, 1993.
} 
d'exagérer, d'omettre, de tromper. Surtout, elles dévoilent comment l'individu, face à ces armes de l'État, est impuissant. Comme dans une tragédie classique, l'humble individu, surtout celui qui montre de l'orgueil en se confrontant aux autorités - Kelly dans Justifying War, Short dans Called to Account - est remis à sa place par les dieux de notre monde laïc, le gouvernement et les médias.

Comme dans tout drame théâtral, les tribunal plays contiennent aussi des changements de ton et de tempo. Norton-Taylor juxtapose le conflit politique avec des détails anecdotiques, afin de fournir une variété dynamique qui maintiendrait l'attention du spectateur. Il inclut des morceaux d'importance apparemment secondaire comme, dans Justifying War, les détails que raconte Mrs Kelly sur les derniers jours avec son mari :

on a dîné. Il est venu avec réticence regarder les infos. À la une il y avait la déclaration que la source avait été révélée. Immédiatement David m'a avoué «c'est moi ». [...] J'ai réagi avec un désarroi total. Mon cœur s'est serré, je m'inquiétais terriblement parce que le fait qu'il m'ait dit ça, je savais là qu'il savait que son nom serait bientôt dans le domaine public. (Norton-Taylor (a), p. 86)

Et dans Called to Account, Short raconte comment elle a bu une tasse de thé avec la femme de Tony Blair, pour lui demander de persuader son mari de ne pas envahir l'Irak. Robin Soans, auteur britannique de pièces verbatim, insiste : "Je n'écris pas une thèse, j'écris une pièce de théâtre » (Soans, p. 39). Ces détails annexes ajoutent de la saveur et de la couleur aux tribunal plays qui, autrement, ne seraient que des comptes rendus journalistiques.

Les tribunal plays adhérent aux conventions théâtrales non seulement en contenant une tension dramatique, une métaphore universelle et un dynamisme rythmique, mais aussi en campant des personnages. Certains auteurs du théâtre verbatim interviewent plusieurs personnes, et en font un amalgame, afin de créer des personnages plus captivants (Soans, p. 42). Kent et Norton-Taylor critiquent cette technique et celle pratiquée par Hare dans Stuff Happens, qui consiste à mélanger témoignages réels et scènes fictionnelles. Kent dit :

La force du théâtre verbatim est qu'il dit toujours la vérité absolue, il contient les mots exacts qu'a dit quelqu'un. [...] Si tout d'un coup tu balances une chose que tu inventes parce que c'est plus facile, je pense que tu défigures la vérité - le résultat se révélerait peut-être très éclairant mais ce n'est pas, à mon avis, la vérité absolue de ce qui s'est passé et de ce qu'on a dit (Kent, p. 153).

Contrairement à certains auteurs de théâtre verbatim, Kent et Norton-Taylor transcrivent de manière aussi littérale que possible les paroles des témoins. Pourtant, en incluant et en excluant certaines parties du discours d'un témoin, Norton-Taylor dessine forcément un portrait spécifique de cet individu. Campbell, après avoir humilié Kelly devant la nation, était l'ennemi public numéro un. Norton-Taylor conclut sa scène avec les mots suivants :

Je voulais juste dire que, comme tout le monde, j'ai été très peiné par le fait que Dr Kelly, qui était clairement quelqu'un de renom, meure de cette manière et évidemment $\mathrm{j}$ 'ai, comme tout le monde j'en suis sûr, réfléchi très longuement aux antécédents de toute cette situation. Donc je pense que tout ce que je dirais, c'est que je trouve sa disparition très, très triste. (NortonTaylor (a), p. 48).

D'une part, ces mots pourraient adoucir Campbell, qui était devenu un paria. D'autre part, le fait qu'il fasse mention du « renom » de Kelly est suspect, étant donné que c'était lui qui avait souligné publiquement son «statut inférieur ». Campbell ne saisit-il pas l'occasion de l'enquête pour s'occuper de sa propre image ? De toutes les façons, ce discours de Campbell 
démontre comment l'inclusion de certains mots par l'auteur ajoute de la complexité et de la couleur au personnage.

Il est donc clair que les auteurs du théâtre verbatim adhérent à la dramaturgie conventionnelle - fait que certains d'entre eux ne nient pas (voir Soans, p. 18 et 41 ; StaffordClark, p. 53 ; Blythe, p. 101-102). Dans Justifying War et Called to Account, les détails de la vie privée de Mrs Kelly et de Clare Short dont j'ai fait mention fournissent le seul répit au déluge de données bureaucratiques. Les auteurs des tribunal plays donnent priorité à la dissémination de l'information factuelle, au-delà de l'intérêt esthétique. C'est sans doute pour cette raison que certaines de leurs pièces ont du mal à maintenir l'intérêt du spectateur.

Le fait que les auteurs des tribunal plays prétendent que leurs pièces présentent au spectateur la réalité sous une forme plus complète, plus honnête et plus pure, est problématique pour de nombreuses raisons. Premièrement, l'idéologie de l'auteur agit sur l'œuvre qu'il écrit. Dans le cas des tribunal plays, le parti-pris de Kent et de Norton-Taylor est clair. À la fin de Called to Account, c'est au public de juger si Tony Blair est coupable ou non. Mais la décision sera-t-elle prise en connaissance de cause ? Kent avoue qu'il était difficile de trouver des témoins prêts à défendre Blair (Kent, p. 144). Même ceux qui paraissent dans la pièce n'ont pas une position tranchée. Perle, conseiller de Rumsfeld, qui avait, avec Bush, mené l'invasion en Irak, avoue qu'avec du recul il ne l'aurait pas soutenu (Norton-Taylor (b), p. 84). Le témoin qui pourrait le mieux défendre Tony Blair - Tony Blair himself ! - n'est même pas représenté au procès. Comment le public pourrait-il formuler un jugement juste? Les tribunal plays, comme toute pièce, sont partiales. D'ailleurs, elles sont souvent polémiques. Il serait donc au mieux naïf, au pire fourbe de la part des auteurs, de prétendre qu'elles sont objectives.

Deuxièmement, les tribunal plays, comme toute autre représentation, sont assujetties aux procès d'esthétisation. Comme je l'ai déjà indiqué, elles sont structurées selon une linéarité aristotélicienne, elles ont des dimensions métaphoriques, elles comprennent des variétés de ton et de rythme, et elles contiennent des personnages ayant une épaisseur. Les tribunal plays ordonnent, manient, truquent, déréalisent la réalité. Le fait que Kent prétende que ses acteurs ne «jouent» pas, est trompeur. Le langage verbatim, comme je l'ai montré avec la citation de Hutton, est souvent très décousu, et demande un acteur expérimenté qui puisse le rendre compréhensible. De plus, comme Kent veut que ses acteurs imitent leur personnage, il est clair que l'artifice esthétique joue un rôle important dans ce théâtre.

Selon Platon, l'artiste est un menteur parce qu'il fait passer son art pour la réalité, alors que cet art n'en est qu'une imitation obscure. On pourrait dire aujourd'hui que ce sont les médias qui mentent, et non l'artiste, parce qu'ils promettent de nous fournir « toute l'histoire », alors que leurs reportages sont souvent tronqués, à sensation, vite consommables et jetables, et assujettis aux intérêts politiques. À leur tour, en prétendant offrir une version plus complète de l'histoire, les tribunal plays, n'obéissent-elles pas à la supercherie des médias ? Georges Lavaudant, metteur en scène français dont le traitement des textes politiques est toujours oblique, onirique et poétique, se répand en injures contre un certain théâtre qui emploie le même réalisme que les médias :

Comme il existe des reality shows à la télévision,

il existe aussi un théâtre qui se

présente, pour user d'une métaphore sportive,

comme capable de marquer l'époque à la culotte.

[...] L'intention

est louable - le résultat désastreux.

C'est la technique violente et irraisonnée

du coup de pied dans la fourmilière. 
Mais est-ce la bonne fourmilière?

Et au bout du compte tous ces coups de

pied spectaculaires et provocateurs deviennent

rapidement ennuyeux et répétitifs.

Ils alimentent à leur manière notre collection

de clichés. Ils ne décollent pas de l'époque,

ils s'y engluent avec complaisance. (Lavaudant, p. 125).

Georges Lavaudant emploie le terme hyperbolique "désastreux », sans doute parce que le théâtre qui suit les actualités au fur et à mesure qu'elles se déroulent - les tribunal plays viennent à l'esprit - remet en question l'idéologie de la Guerre contre le terrorisme (dont il fait mention dans la même communication) sans pour autant mettre en cause la forme réaliste, apparemment transparente, dans laquelle elle est présentée. La " bonne fourmilière » dans laquelle ce théâtre devrait donner un " coup de pied », serait l'évidence trompeuse, la « collection de clichés » de la forme réaliste utilisée par les médias.

Les tribunal plays, tout comme les médias, lissent la distance entre réalité et représentation. Elles cachent l'artifice de leur propre composition, brouillant ainsi la distinction entre réalisme et réalité, et créant un continuum fusionnel qui fait écho aux médias, au lieu de les contester. Ce n'est pas le cas du théâtre verbatim. L'Instruction de Weiss, ainsi que le 11 septembre 2001 de Michel Vinaver, dont la forme "se rapproche, selon l'auteur, de celle des cantates et des oratorios, se composant d'airs (à une, deux ou trois voix), de parties chorales » (Vinaver, p. 9), affichent de façon consciente la partialité, la subjectivité, la partie esthétique de la représentation théâtrale. À mon avis, l'auteur politique endosse la responsabilité de montrer de manière explicite que sa version des événements est théâtrale, subjective, artistique, et surtout incomplète.

Troisièmement, les tribunal plays ne fournissent au spectateur guère plus d'informations que la lecture des bons journaux. Les détails inclus dans Justifying War et dans Called to Account sont connus par le public intellectuel de gauche du Tricycle. Georges Lavaudant avertit qu'une représentation réaliste des actualités court le risque d'être « ennuyeu[se] et répétiti[ve]», ce qui est parfois le cas avec les tribunal plays. Il est vrai, comme je l'ai expliqué, qu'elles contiennent certains détails personnels intéressants. Mais en fait, ces détails aussi les rapprochent du journalisme au lieu de les en éloigner. Georges Lavaudant critique l'exploitation par les médias de la souffrance :

La télévision nous gave d'images brutes, violentes, insoutenables.

"C'est horrible» murmurons-nous. Mais nous en redemandons. On veut du 11 septembre.

On veut de la Tchétchénie. On veut les cris, les hurlements, le sang. (Lavaudant, 134).

Justifying War se termine avec les mots de Mrs Kelly, qui conjure : " puis-je prendre cette opportunité pour demander aux médias de continuer à respecter le désir de ma famille qu'ils préservent notre vie privée ?» (Norton-Taylor (a), p. 95). Paradoxalement, la même scène fait étalage du grief de Mrs Kelly et des détails sinistres du suicide de son mari : il prend une surdose du médicament anti-arthrose de sa femme, et s'ouvre les veines. Cette révélation, comment diffère-t-elle du voyeurisme caractéristique des médias?

L'intention des tribunal plays est digne de louanges, certes. Elles militent contre l'incompétence, la négligence et la malhonnêteté de ceux qui sont au pouvoir, et de ceux qui parlent du pouvoir dans les médias. Selon David Lescot et Laurent Véray, directeurs du recueil d'essais Les Mises en scène de la guerre au XX siècle, « la guerre bouleverse les conventions, les canons génériques, pousse les metteurs en scène à inventer d'autres moyens pour produire d'autres effets dans le champ de l'art théâtral » (Lescot et Véray, p. 7). À la 
suite des attentats du 11 septembre 2001 et de la Guerre contre le terrorisme, les créateurs des tribunal plays - genre théâtral de plus en plus courant en Grande-Bretagne - ont conçu une nouvelle esthétique théâtrale. L'auteur, ainsi que le metteur en scène, adoptent une esthétique hyper-naturaliste, estimant qu'elle rendra avec objectivité et clarté les événements qui sont souvent obscurcis dans les mondes de la politique et des médias. Pourtant, je suis d'avis que, avec leurs vaines promesses de véracité et d'exhaustivité, les tribunal plays se rapprochent des médias plus qu'elles ne s'en éloignent. Il est du domaine particulier de l'art de reconnaître que toute information est partielle, incomplète, subjective. De cette incapacité de tout comprendre, de tout écrire et de tout jouer, naissent de nouvelles manières de concevoir la politique, la scène et le monde. 


\section{Bibliographie}

Aristote, La Poétique (Paris : Poche, 1990).

Billington, Michael, State of the Nation: British Theatre Since 1945 (Londres : Faber, 2007).

Blythe, Alecky, in Hammond, Will and Steward, Dan (dir.), Verbatim verbatim: Contemporary Documentary Theatre (Londres : Oberon, 2008).

Finburgh, Clare, "Le théâtre britannique contemporain post-11 septembre 2001 : du théâtre verbatim à l'allégorie ", in Lescot, David et Véray, Laurent (dir.), Les Mises en scène de la guerre au XX siècle (Paris : Nouveau Monde, 2001).

«La Théâtralisation de l'attentat: la représentation du terrorisme dans le théâtre britannique contemporain ", in Christine Douxami (dir.), Théâtres politiques (en) Mouvement(s) (Besançon : Presses universitaires de Franche-Comté, 2011).

Hare, David, in Hammond, Will and Steward, Dan (dir.), Verbatim verbatim: Contemporary Documentary Theatre (Londres : Oberon, 2008).

Kent, Nicolas, in Hammond, Will and Steward, Dan (dir.), Verbatim verbatim: Contemporary Documentary Theatre (Londres : Oberon, 2008).

Larthomas, Pierre, Le Langage dramatique (Paris : Armand Colin, 1972).

Lavaudant, Georges "Considérations intempestives", in Mises en scène $d u$ monde (Besançon : Les Solitaires intempestifs, 2005).

Lescot, David et Véray, Laurent (dir.), Les Mises en scène de la guerre au XX siècle (Paris : Nouveau Monde, 2001).

Norton-Taylor, Richard (a), Justifying War: Scenes from the Hutton Inquiry, (Londres : Oberon, 2004).

(b) Called to Account: The Indictment of Anthony Charles Lynton Blair for the Crime of Aggression Against Iraq - A Hearing (Londres : Oberon, 2007)

(c) in Hammond, Will and Steward, Dan (dir.), Verbatim verbatim: Contemporary Documentary Theatre (Londres : Oberon, 2008).

Picon-Vallin Béatrice, "La Scène comme utopie », in Mises en scène du monde (Besançon : Les Solitaires intempestifs, 2005).

Ravenhill, Mark Product, in The Cut and Product (Londres : Methuen, 2006).

Scherer, Jacques et Scherer, C., Le Théâtre classique (Paris : Presses Universitaires de France, 1993).

Soans, Robin, in Hammond, Will and Steward, Dan (dir.), Verbatim verbatim: Contemporary Documentary Theatre (Londres: Oberon, 2008).

Stafford-Clark, Max, in Hammond, Will and Steward, Dan (dir.), Verbatim verbatim: Contemporary Documentary Theatre (Londres: Oberon, 2008).

Vinaver, Michel, 11 septembre 2001 (Paris : L'Arche, 2002)

Weiss, Peter, L'Instruction (Paris : L'Arche, 2000).

Williams, Roy, Days of Significance (Londres : Methuen, 2007). 
À propos de l'auteur :

Clare Finburgh est maître de conférences en théâtre moderne à l'Université d'Essex en Angleterre. Son domaine de recherches porte essentiellement sur les théâtres moderne et contemporain britannique, français et francophone. Elle a coédité un recueil d'essais sur le théâtre et la politique de Jean Genet intitulé Genet: Performance and Politics (Palgrave Macmillan, 2006), ainsi que Contemporary French Theatre and Performance (Palgrave Macmillan, 2011), et elle a coécrit avec David Bradby un livre sur la mise en scène du même auteur (Routledge, 2011). Elle prépare actuellement une monographie sur la représentation de la guerre et du terrorisme dans le théâtre britannique depuis 2001.

Pour citer ce document :

Clare Finburgh, «« Vérité » ou «mensonge »? Le Théâtre politique post-11 septembre en Grande-Bretagne», Agôn [En ligne], Dossiers, HS n 1 : Mettre en scène l'événement, Mémoire de l'événement : le cas du 11 septembre au théâtre, mis à jour le : 08/09/2011, URL : http://agon.ens-lyon.fr/index.php?id=1808. 